\title{
Reply to Comment on the paper " On a role of quadruple component of magnetic field in defining solar activity in grand cycles" by Usoskin (2017)
}

\author{
Zharkova V. ${ }^{\text {a }}$, Popova E. ${ }^{\text {b }}$, Shepherd S.c, Zharkov S. ${ }^{\text {d }}$ \\ ${ }^{a}$ Northumbria University, Department of Mathematics, Physics and Electical \\ Engineering, Newcastle, UK \\ ${ }^{b 1}$ Federal State Budget Educational Institution of Higher Education M.V.Lomonosov \\ Moscow State University, Skobeltsyn Institute of Nuclear Physics, ${ }^{2}$ Schmidt Institute of \\ Physics of the Earth of theRussian Academy of Sciences, Russia \\ ${ }^{c}$ Bradford University, School of Engineering, UK \\ ${ }^{d}$ Hull University, Department of Physics and Mathematics, Kingston upon Hull, UK
}

\begin{abstract}
In this communication we provide our answers to the comments by Usoskin (2017) on our recent paper (Popova et al, 2017a). We show that Principal Component Analysis (PCA) allows us to derive eigen vectors with eigen values assigned to variance of solar magnetic field waves from full disk solar magnetograms obtained in cycles 21-23 which came in pairs. The current paper (Popova et al, 2017a) adds the second pair of magnetic waves generated by quadruple magnetic sources. This allows us to recover a centennial cycle, in addition to the grand cycle, and to produce a closer fit to the solar and terrestrial activity features in the past millennium.
\end{abstract}

Keywords: Solar activity; principal components; dynamo waves; Gleissberg cycle

\section{Introduction}

In this communication we provide answers to the comments by Dr. Usoskin (Usoskin, 2017, referred hereafter as U17) to our paper (Popova et al, 2017a, referred hereafter as PZSZ17). U17 critisizes the methods used in PZSZ17, the validation of the results of our previous papers (Shepherd et al, 2014; Zharkova et al, 2015) and disputes the results obained by PZSZ17. In this 
reply we show that the methods used by us are correct for the oscillatory function as the solar activity curve is and the results are well validated by a comparison with known sunspot observations. Moreover, we show that own papers of U17 contain the evidences, which undoubtedly confirm our predictions.

Below we elaborate on these statements by replying to the comments in turn.

\title{
2. Method
}

1. Comment 1. In the first line of the comments U17 states that 'the prediction method in PZSZ17 is based on a simple three-harmonic model of solar activity (two dipole and one quadruple components) predicting solar activity for 3000 years.

\begin{abstract}
Answer.
This is not a correct statement. At first, PZSZ17 did not derive the summary curve from a dynamo model, but used the analytical expressions for the two principal components and their summary curve derived from the magnetic field observations by Wilcox Solar Observatory (Shepherd et al, 2014). Only later (Zharkova et al, 2015) this curve was assigned to two waves generated by a solar dynamo in two layers by dipole magnetic sources. In PZSZ17 the summary curve of these two waves was added with a quadruple wave component with parameters of the second pair of eigen vectors derived from the observations (Zharkova et al, 2012). At second, PZSZ17 prediction is made only for 2000 years (forward by 1200 years and backward by 800 years). The prediction for 3000 years is made in another paper (Zharkova et al, 2017) without using a quadruple component.
\end{abstract}

Below we eleborate on the description of these points.

The first prediction of solar activity for 2000 years was done by us in the previous paper (Zharkova et al, 2015) with the summary curve plotted in Fig.3 using the method described by Shepherd et al (2014) (their Figs.3 and 4). It is clearly seen that this curve for 2000 years is made only from the observational data derived with Principal Component 

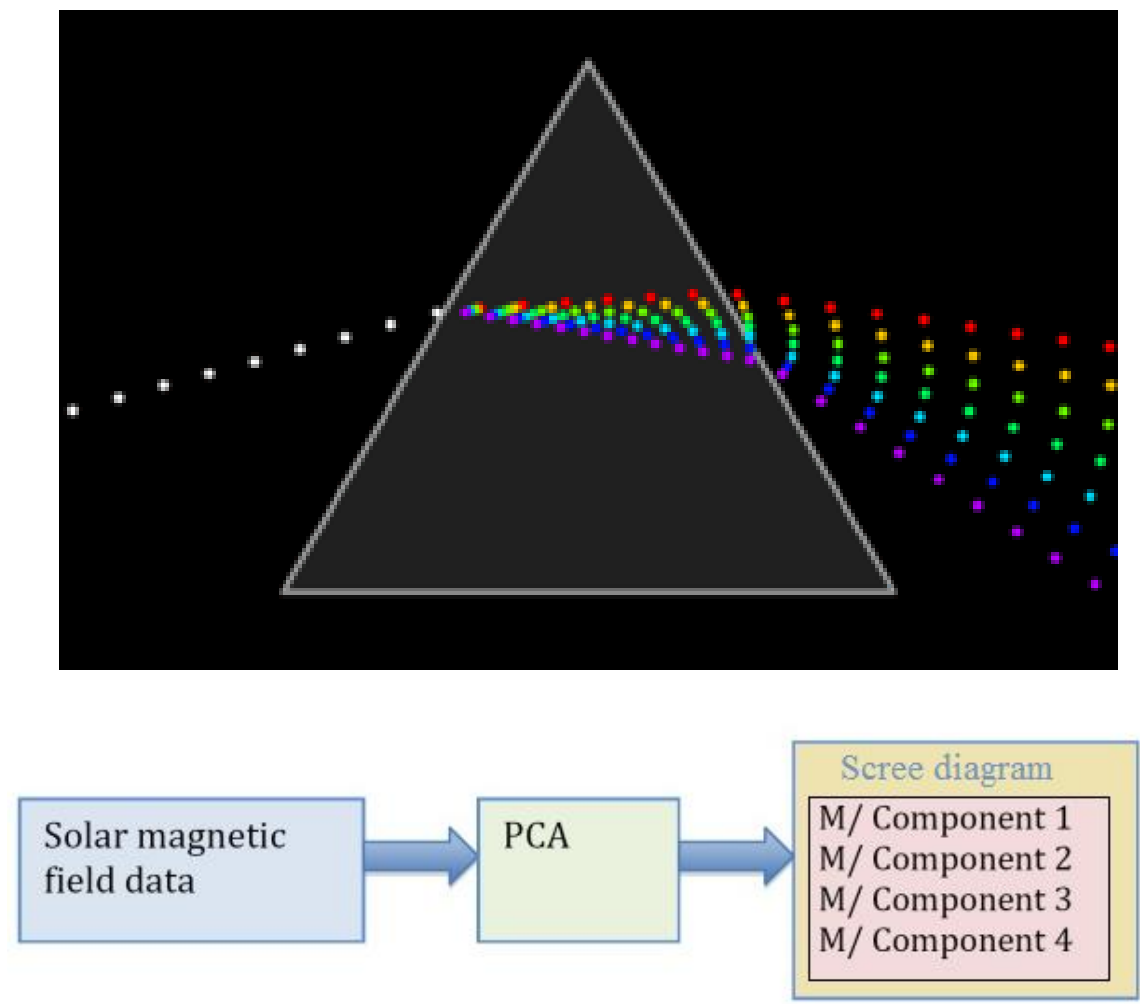

Figure 1: Top plot: schematic cartoon showing a glass prism decomposing the electromagnetic wave of white light (left) into the waves with different wavelegth (from red to ultra-violate) (right). Bottom plot: schematic representation of Principal Component Analysis (PCA) acting as the similar filter for decomposing the solar magnetic wave seen on the solar surface into separate eigen vectors, or magnetic waves with their own eigen values describing separate physical processes (Zharkova et al, 2012). 
Analysis (PCA) and symbolic regression analysis without involving any dynamo models as we clearly indicated in the papers (Shepherd et al, 2014; Zharkova et al, 2015).

Similar to a glass prism (see Fig.1, top plot) used to decompose white light into the waves with different wavelengths: from ultraviolet, blue to orange and red, we applied PCA (Zharkova et al, 2012) as a similar filter to the whole disk magnetograms obtained by Wilcox Solar Observatory (see Fig.1, bottom plot) to decompose eigen values and eigen vectors of the individual magnetic waves from a whole variety of waves present in the magnetic field data. The PCA was introduced in 1901 (Jolliffe, 2002) and applied before and after us to the solar background magnetic field of the same WIlcox Solar Observatory (see for details Lawrence et al, 2004; Cadavid et al., 2005, 2008), or for deriving activity patterns in the solar wind (Holappa et al, 2014).

The PCA method (Jolliffe, 2002) uses the covariance matrix of the magnetic field data and its variance as the eigen values of magnetic oscillations defining the magnetic wave properties as shown in our Scree plot (see Fig.1 in Zharkova et al (2012). Using PCA, we managed to derive the eigen values of own solar magnetic oscillations (see Scree diagram in Fig.2) showing clearly, at least, 4 noticeable pairs with large eigen values covering $96 \%$ of the total data variance. The first pair is called Principal Components (PCs) of magnetic waves, which are defined by magnetic data with the total variance of $39 \%$ (Y-axis) corresponding to $67 \%$ data by standard deviation.

The first two highest eigen values were used to built eigen vectors for the two principal components, or coherent magnetic waves, corresponding to these eigen values (see Fig. 2 and 3 in Zharkova et al, 2012). This is equavalent to deriving different wavelengths of white light, after it is split in a prism (see Fig.1). Now these two magnetic waves can be assigned to unique physical processes (Jolliffe, 2002). By applying Parker's model for two layers with meridional circulation (Parker, 1993), we derived that these PC waves correspond very closely to the waves generated by dipole magnetic sources (Zharkova et al, 2015).

However, we did not only find the two principal components (Zharkova 


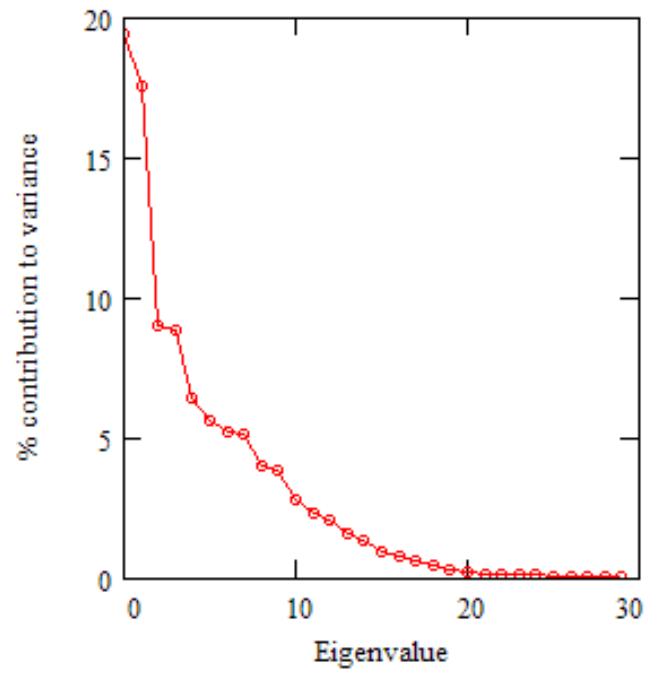

Figure 2: The Scree plot decomposing the magnetic waves present in full disk magnetograms into the pairs of eigen values defining the magnetic wave eigen vectors sorted by their variance (reproduced from Fig.1 of Zharkova et al (2012)).

et al, 2012), but also using the symbolic regression analysis with Hamiltonian approach (Schmidt and Lipson, 2009) derived the symbolic description of these two principal components, derived purely from the solar magnetic field observations (Shepherd et al, 2014). Using these symbolic representations of $2 \mathrm{PC}$ curves and the coefficients defining these curves, we also derived their summary curve and its coefficients (Zharkova et al, 2015).

This analytical summary curve was then used to calculate magnetic waves for 2000 years (Zharkova et al, 2015). The calculation is a very lengthy process in desktop PC, this is why originally we calculated only the curves by a millennium backward and forward. Now our calculations are extended to 3000 years (back from the present timet o 1000 BC) (Zharkova et al, 2017). We are also working on the calculation back to 100000 years and hope to present the extended summary curve soon.

We clearly indicated that PCA is a precise method (Jolliffe, 2002). Hence, PCA accuracy is defined only by the accuracy of the original 
measurements of background magnetic field, which is rather high in the ground-based observations, compared to the accuracy of measuring sunspot areas. The accuracy of calculations was discussed in our papers above (Shepherd et al, 2014; Zharkova et al, 2015). We demonstrated by testing PCs and their summary curve derived for cycle 21-23 for prediction of cycle 24 that revealed the accuracy above $97.5 \%$ (see Fig. 2 in Shepherd et al, 2014). We also compared our summary curve with averaged sunspot numbers presented in Fig. 3 showing their close correspondence. Recently, the accuracy of our prediction of solar activity was confirmed (Zharkova et al, 2017) by the comparison with the observations of sunspots in 14-15 century with a naked eye, when they were available (see Fig.4 here from Zharkova et al, 2017).

Note from the above description, the 2 PCs and their summary curve are calculated without any assistance of dynamo models, but using only the WSO magnetic field observations. Only later (Zharkova et al, 2015) for the first time we included modelling of PCs as dynamo waves generated by dipole magnetic sources using Parker's modified dynamo model for two layers meridional circulation (Parker, 1993). This was done to prove that the 2 PCs are, in fact, the magnetic dynamo waves produced by dipole magnetic sources in two layers of the Sun: one at the bottom of the solar convective zone and the other close to the surface. The simulated dynamo waves are found to fit very closely the PCs derived from the observations (see Figs. 3 and 6 in Zharkova et al, 2015). Curiously enough, the helioseismic observations by SDO confirmed the existence of these two layers (or super cells) in each hemisohere (Zhao et al, 2013). The similar finding was confirmed by the helioseicmic observations by GONG as well (Jackiewicz et al, 2015).

However, besides these 2 PCs, in the Scree plot (Fig.2) there are another 3 pairs of meaningful eigen values, which are assigned to another physical processes. The latitudinal magnetic waves corresponding to the first four pairs were plotted in Fig. 5 (Zharkova et al, 2012) clearly showing that the maximal amplitudes of the waves of the second pair is (30-40)\% lower than of the first pair and of the third pair (30-40)\% lower than of the second pair and so on. Moreover, the cross-correlation analysis of these 8 components shown in Fig.6 of Zharkova et al (2012) and reproduced here in Fig.5, clearly shows that cross-correlation of the 
two principal components reveals for sure dipole sources (see the top left plot), while the cross-correlation of the pairs 2 and 3 (the top right and bottom left plots, respectively) clearly reveal quadruple and sextuple sources. These findings (Zharkova et al, 2012) were used by Popova et al (2013) to show that the secondary latitudinal waves derived for the other two pairs of magnetic waves in cycles 21-23 are best represented by the dynamo waves produced by quadruple magnetic sources.

2. Comment 2. U17 admits that in PZSZ17 the dipole components are periodic functions with frequencies being close to each other (21.41 and 22.62 years), which leads to a beating frequency of about $350-400$ years, and were defined elsewhere (Zharkova et al, 2015) from a 35-year long set of solar data. However, U17 states that as known from data processing, frequencies cannot be defined with the necessary precision from this dataset. For example, in order to separate, in a statistically significant way, these two frequencies, one needs about 400 years of data. Therefore, the beating period of 400 years can not be accurately defined from such a short dataset and is a pure artefact, which cannot be statistically defined from the available data.

The quadruple component is introduced as a purely ad-hoc sine wave with the period chosen to obtain the third beating period of around 100 years. Thus effectively, the authors of PZSZ17 represent the long-term solar activity by a multi-harmonic oscillator. This approach would work only for a precisely known and purely stationary series. However, this is clearly not a case for solar activity which contains an essential intrinsic chaotic/stochastic component (e.g., (e.g. Kremliovsky, 1995; Petrovay, 2010; Usoskin, 2008). Similar attempts to model solar variability by a multi-harmonic (also nonlinear) oscillator have been preformed since the 1950s (see e.g. Parker, 1955) but failed.

\section{Answer \\ We understand that U17 and other authors listed in the paper struggled to derive any frequencies from the sunspot data because they contain a combination of all the magnetic waves produced by the Sun (being a white light equavalent). Of course, it is not possible to reproduce the white light, which does not have a wavelegth, by a wave with a single wavelegth (either red or UV light). This point of short dynamo}



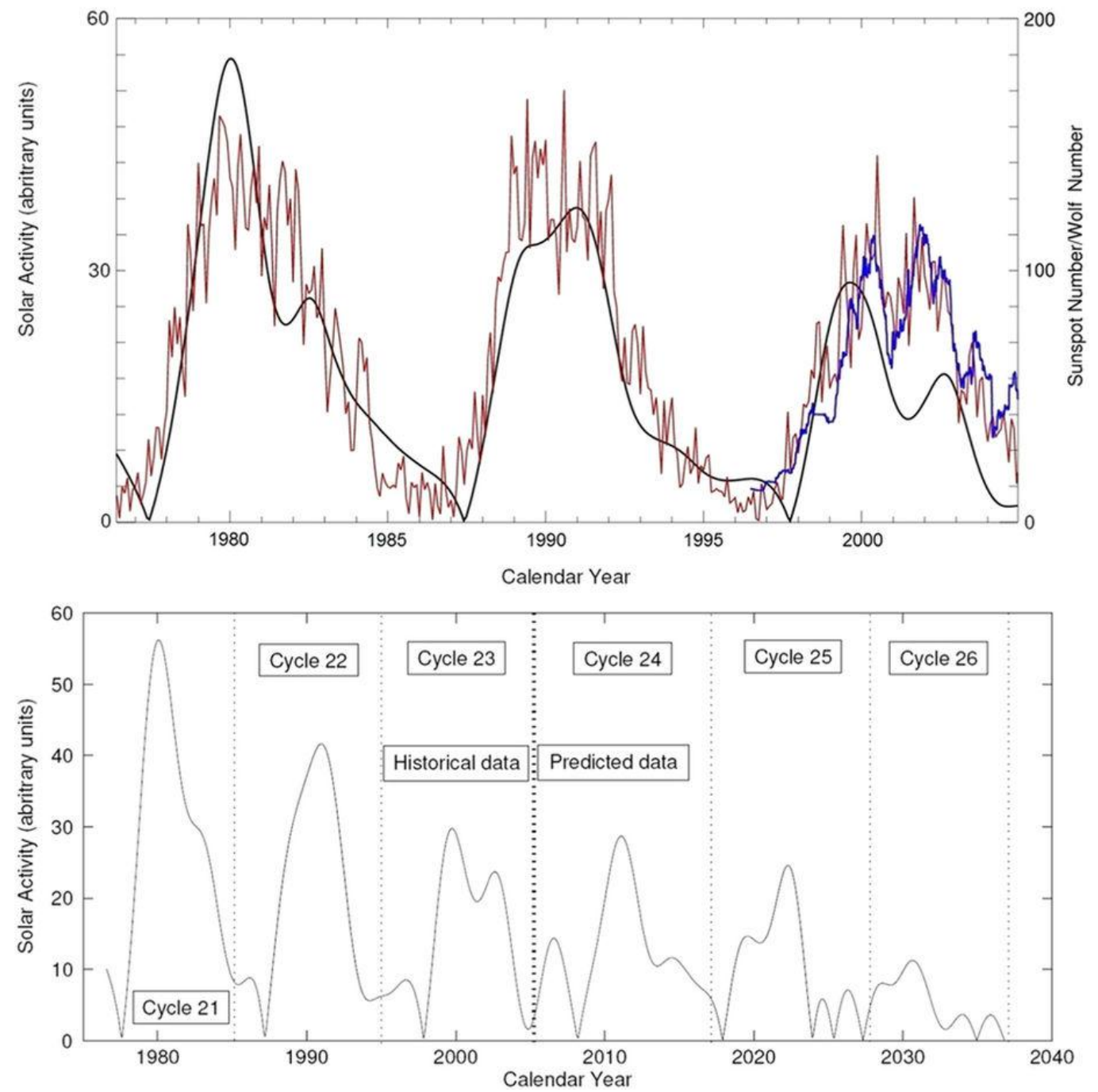

Figure 3: Fitting our modulus summary curve to the averaged sunspot numbers for cycles 21-23 (top plot) and the prediction of sunspot numbers for cycles 24-26 (bottom plot) (from (Zharkova et al, 2015)). 


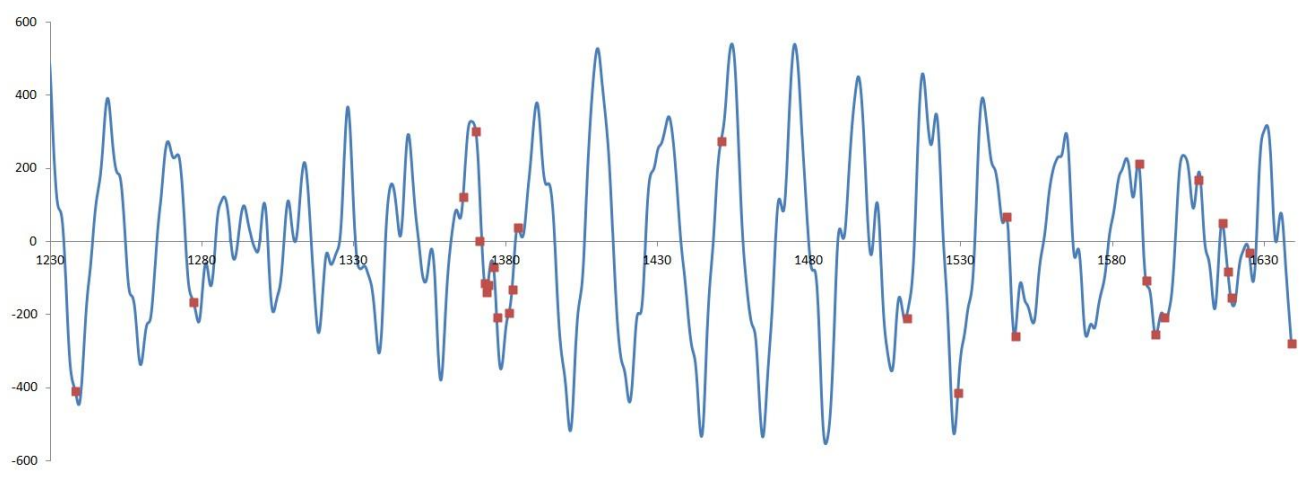

Figure 4: Summary curve (blue line) predicting solar activity in the past grand cycle (1317 centuries) showing the naked eye observations of sunspot in the pre-telescope era (red dot) when they were available (from (Zharkova et al, 2017))

memory was recently reported by Karak and Nandy (2012). This is why one needs a PCA to uncover the components of magnetic waves (see answer A1). Parker recognised the problems with his first model (Parker, 1955) and introduced a dynamo model for two layers (Parker, 1993), which we also utilise in our model.

Moreover, the magnetic waves reflected in occurences of sunspots are, in fact, those of the solar toroidal field (embeded into the photosphere as sunspots), and not of the poloidal field reproduced by us. We tried PCA for sunspot data in cycle 23 and discovered only the activity belts where they occur (Zharkova et al, 2012). This is because sunspot areas are much smaller, thus, the definition of any waves with sunspots have much larger error bars than those derived from the background magnetic field using the areas from the latitudinal strips of the whole solar disk (Zharkova et al, 2012).

Hence, despite the statement by U17, our research (Zharkova et al, 2012; Shepherd et al, 2014; Zharkova et al, 2015) has proven that if the correct data and method are used then 'the frequencies can be defined with the necessary precision, and this has been done from the different dataset, e.g. from the solar background magnetic field'. These results are shown (Shepherd et al, 2014; Zharkova et al, 2015) to be very stable 

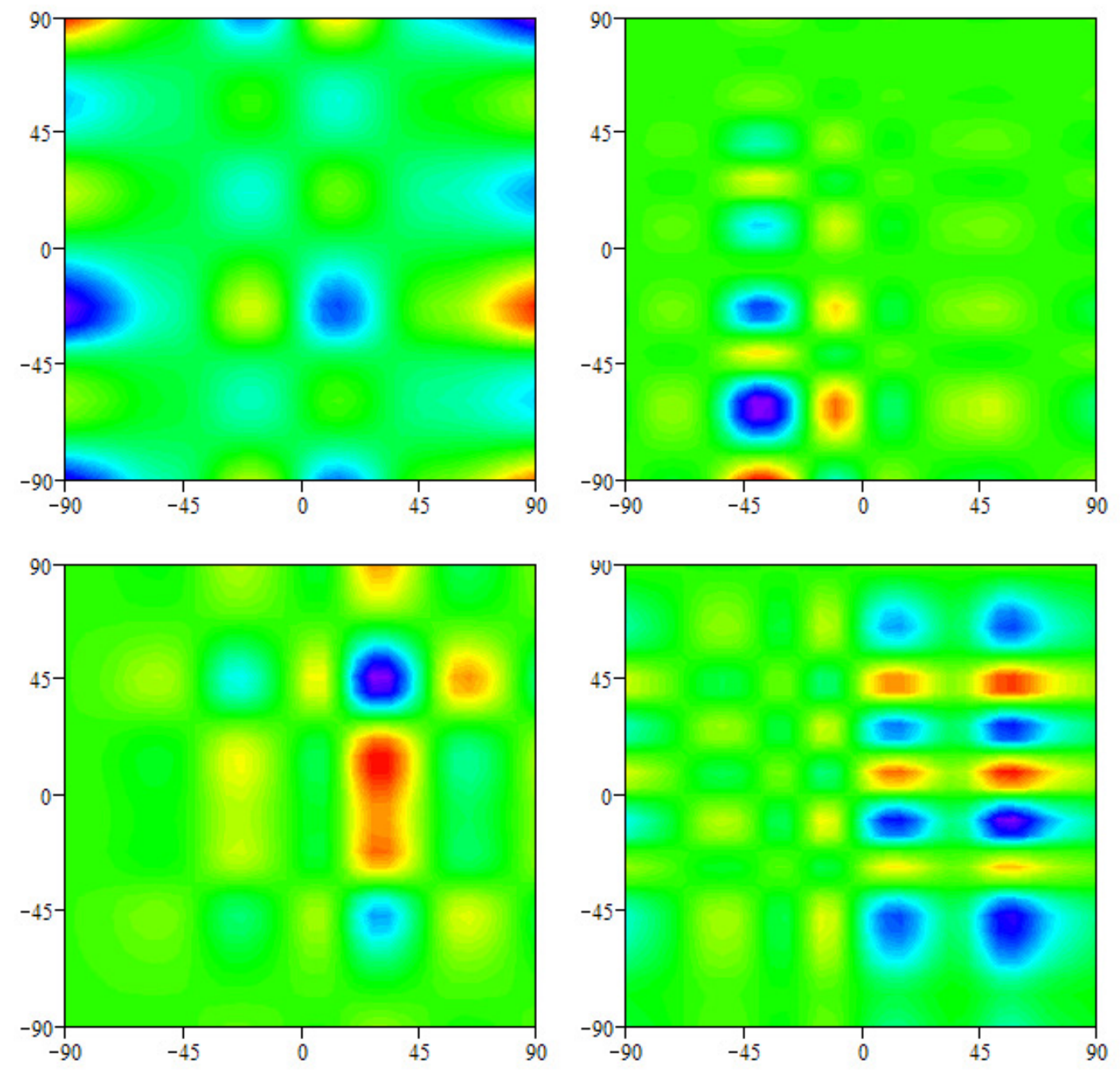

Figure 5: The cross-correlation between the four pairs of the latitudinal solar magnetic field variations shown in Fig. 5 ((Zharkova et al, 2012) over the 30 solar latitudes corresponding to the pair of principal components (top left plot) and pair 2 (top right plot), pair 3 (bottom left plot) and pair 4 (bottom right plot) from the Scree plot shown in Fig.1 of Zharkova et al (2012). 
and to keep their eigen values and eigen vectors (and thus, frequencies) independently from the length of the data derived from: either for any single cycle from three cycles considered (21-23), or for any combination of pairs of cycles from these 3 cycles, or for all 3 of them. This is similar to a human ECG test taken within ten minutes to discover the rythm of a human heart. If this rythm has stochastic component this is the indication of a heart desease. This PCA of the solar magnetic field heartbeat proves that the Sun's heartbeat, or dynamo machine, works tirelessly and steadily producing the waves with the same very close frequencies over thousands (and may be billions) of years indicating that the Sun is in a very good 'stellar health'. If the Sun's dynamo wave generation would be stochastic as suggested by U17, we should be worried about its possible explosion soon. Luckily, we believe, there are no any signs for these worries.

Historically, many researchers, including U17, were trying to derive solar activity from sunspots, which are secondary products of the solar dynamo machine with its main product being a poloidal magnetic field produced at the bottom of the solar convective zone (Parker, 1955, 1993; Jones etal., 2010). This poloidal field is the one, which allows magnetic flux tube (which become sunspots on the surface) to move through the solar interior owing to magnetic buyancy and $\alpha$-effect, while differential rotation to convert this magnetic field into the toroidal field of magnetic flux tubes, or sunspots (see review by Jones etal., 2010, and references therein). Therefore, strictly speaking, researchers using sunspots to describe the solar activity governed by solar dynamo, try to define the variations of poloidal magnetic field from the measurements of the toroidal one. And given the fact that on the solar surface a superposition of many magnetic waves is observed, it is not possible, indeed, to predict with a single dynamo model their behavior over the period longer than half of a solar cycle (see for example Karak and Nandy, 2012) unless using the solar magnetic field near poles (Choudhuri et al, 2007), which is a part of the poloidal field used in our approach.

In the case of the PCA and symbolic regression approach presented by us, the matter was significantly simplified: 1) we use the appropriate data - solar background magnetic field - instead of sunspot numbers giving large errors because of their small areas covered by them com- 
pared to the whole solar disk area; 2) we derive with PCA the exact eigen values and eigen vectors of solar magnetic field oscillations; 3) we assign separate physical processes to each eigen vectors derived from this background magnetic field and 4) we check the outcome with the relevant simulations using these mechanisms. The results obtained with such the approach are simply overwhelming, because they allow us to discover that 1) the magnetic waves in the Sun are generated in pairs (see Fig.2), in the inner and outer solar interior; 2) the main pair, or principal components, are now very well defined by Parker's two layer dynamo model developed for dipole magnetic sources (Parker, 1993; Zharkova et al, 2015); 3) there are other 3 pairs of waves which are also generated on the Sun (see Scree plot in Fig.2).

The periods of the two main waves (21.41 and 22.62) generated by a dipole discovered with PCA are given to us directly by the eigen values and eigen vectors derived by PCA, as it is a precise method. This period comes from addition of cosine functions with different arguments as described by formula (1) in section 'Beating effects' in Zharkova et al (2015) derived from a basic mathematical formula of summation of cosine functions with different arguments. After we got these PC waves shown in Fig.1 in (Zharkova et al, 2015), we automatically obtain their periods presented. Readers can experiment with diferent periods to discover that only this combination would fit the observational curves of the two PCs derived by Zharkova et al (2015). The rest was the matter of a pure arithmetical exercise to derive the exact period and dynamo numbers allowing us to reproduce these curves with Parker's two layer dynamo model.

The main point was that the phase between these two waves is also described by a periodic cosine function, which brings some level of stochasticity when calculating the wave interference and the summary curve. These periods and phase shifts naturally led us to the grand cycles of slightly different lengths appearing owing to the beating effects of these two waves with the slightly variable periods, depending on the frequencies of the waves and phase shifts in each particular grand cycle (see Fig.3 in Zharkova et al (2015) or Fig.2 in PZSZ17). Although, unlike Choudhuri et al (2012), we did not need to introduce any stochasticity to solar dynamo waves produced by dipole magnetic 
sources, as the $\mathrm{PC}$ waves have it is already incorporated into formulae of the summary curve with $\cos (\cos )$ dependence of their phases.

The further element of visible stochasticity of magnetic field variations is now handled by adding the components of magnetic waves produced not only by dipole but also by quadruple magnetic sources as shown in the paper PZSZ17. The presence of quadruple magnetic sources in magnetic field observations was shown from cross-correlation of relevant independent components for 4 pairs derived with PCA in our previous paper (Zharkova et al, 2012) represented here in Fig.5 (see also answer A1). Note that the period of a quadruple wave added by us was not 100 years as U17 states, but it was equal to 27.24 years as clearly described in section 4.1 of PZSZ17. 100 year period appears naturally as the period of the envelope wave caused by a beating effect between dipole and quadruple waves.

PZSZ17 shows that the beating effect of the three waves will produce two beating effects, or two envelope curves: a grand cycle of about 400 years and the centennial cycle of 100 years as shown in Figs.2 and 3 in PZSZ17. Evidently, to obtain the centennial period of 80 , or 90 years we would need slightly (by a unit or so) to amend the period of a quadruple wave done in Popova et al (2017b). We hope to derive soon from the magnetic field data the analytical expressions for real eigen vectors of the second pair of independent components from Scree plot, that will allow us to incorporate the real periods of quadruple waves during each grand cycle, which seem to have different lengths. We are confident that they will be close to that we selected in the current paper, because according to PCA, each eigen vector describes a separate process, which for the pair two in Scree plot corresponds to quadruple waves.

However, we understand the problems, including usage of a single oscillator faced by U17 and the other authors from the lists of the papers presented in the comment. As we have shown above (see answer A1), there is no a single oscillator in the Sun, there are superposition of different (about 5 in each layer) waves produced by different magnetic sources. The PC waves are produced by dipole sources located in two layers of the interior (see answer A1). In fact, using the sunspot data 
alonet he other authors could not predict the solar activity curve for a long period (see references in Introduction of Zharkova et al, 2015). This is because the sunspot solar activity curve is an oscilatory (periodic) function while the authors applied to them a linear regression analysis developed for normal data (having standard Gaussian probability density function and straightforward cumulative function) not applicable to a periodic function.

Following the theory of linear regression analysis (see, for example, Seber, 2012, and references therein) there are four principal assumptions, which justify the use of linear regression models for purposes of inference or prediction (see also https://en.wikipedia.org/wiki/Linear_regression). The data have to be rigorously tested to satisfy the conditions where this method is applicable, e.g tested for normality using KolmogorovSmirnov test, at least. If any of these assumptions are violated, then the forecasts, confidence intervals, and scientific insights yielded by a regression model may be (at best) inefficient or (at worst) seriously biased or misleading.

Hence, if the original data are showing a periodic function then the periodic regression analysis must be used. These could be sin or cos functions fitted to the periodic function when the underlying mechanism generating the periodicity is an actual sine or cosine function. In those cases, you can transform the data with an arcsine transformation and proceed with the traditional linear regression (see examples on http://iase-web.org/documents/papers/icots7/C207.pdf, http://www.ct.gov/caes/lib/caes/documents/publications/bulletins/

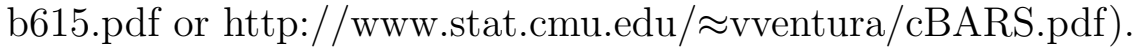

Moreover, the cycle variables for solar activity are not fully independent because a magnetic field of the future cycle appears half way through in the current cycle (Choudhuri et al, 2007), showing, in fact, the data in two cycles being clearly related each to other and not independent as required by the statistical method. In this case, the other methods of fitting (Bayesian, ANN or Principal Component Analysis (PCA), etc) are more appropriate as many authors have shown (see for example Lawrence et al, 2004; Cadavid et al., 2005, 2008; Zharkova et al, 2012). PCA is a well known precise method, which allows researchers 
to decompose a complex function into simpler components, which can be assigned to separate physical processes (see Zharkova et al, 2012, and references therein).

Visibly periodic variations of the observed solar magnetic field with highest variance derived with PCA then can be interpreted by a set of periodic functions using non-linear periodic regression with Hamiltonian analysis (Schmidt and Lipson, 2009) as applied in the analysis by (Zharkova et al, 2015). This, we believe, is the correct interpretation of a complex series of solar activity, which helps researchers to uncover its secrets.

3. Comment 3. U17 wrote that PZSZ17 do not present any analysis of the stability and robustness of the method and provide no clue on the range of its validity.'

\section{Answer}

This is clearly a misleading statement. PZSZ17 does not present a new method, but uses the findings of the previous three papers published in MNRAS (Zharkova et al, 2012), Astrophysical Journal (Shepherd et al, 2014) and Nature Scientific reports (Zharkova et al, 2015). In PZSZ17 we clearly refer to these papers in the relevant points. Now we wish to direct the readers to the answer A1 where the details of the method accuracy (97.5\%) is discussed with the references to these papers. Obviously, U17 is familiar with all the papers because the similar comments were also placed (and answered by us) below the paper (Zharkova et al, 2015) (see comments A1 above).

4. Comment 4. U17 states that PZSZ17's choice of the main beating frequencies is ungrounded and imprecise. For example, the Gleissberg cycle is not a single 100-yr mode but rather a wide-band variability with typically two sub-modes, 70-90 years and 120-150 years (e.g. Ogurtsov et al, 2002; Vecchio et al, 2017).

\section{Answer}

This statement about beating frequencies for Gleissberg cycle is partially valid, its periods can vary for different times. We only considered the one of 100 years relevant for the past grand cycle. The variety 
of Gleissberg periods (80-120 years) can be real, similar to different lengths of the grand cycles discovered by Zharkova et al (2015). We are working to get these periods and symbolic description of these quadruple waves found as the second pair of waves in the Scree plot (see Answer A1).

But so far we were unable to derive the symbolic function for this pair (because of lack of time, not because its impossibility) and decided to use the one modelled by dynamo equations. Since we were targeting two centennial minima in the 19 and 20 centuries seen in the past grand cycle, where this period was about 100 years, we used the period of quadruple wave of 27.42 year. This is the only period number allowing us to produce the additional minima of the centinneal cycle of 100 years via a triple beating effect by adding it to the periods of the two waves found from the principal components. For other grand cycles the period of quadruple waves defining Gleissberg cycle, can slightly vary by a few digits after the decimal dot. These variations easily lead to the Gleisberg cycle to vary from 80 to 130 years reported in another paper (Popova et al, 2017b).

The different periods can be real because PCA shows in the Scree plot that, besides of $2 \mathrm{PCs}$, there are other two pairs of magnetic waves, with the total eigen values of about $19 \%$ of total variance. If the two PCs (magnetic field waves) are considered as equivalent of ultra violate waves of white light, these next two magnetic waves can be as a blue component obtained from white light (or the total magnetic field).

5. Comment 5. U17 wrote that the 400-year cycle claimed by PZSZ17 is not pronounced in solar activity. Instead, the very well-defined Suess / de Vries cycle of 210 - 240 year periodicity is not present here.

\section{Answer \\ We are surprised with this statement. First, the curve in Fig.3 (Zharkova et al, 2015), Fig. 3 in PZSZ17 and Fig.1 (Zharkova et al, 2017) clearly show the grand cycle fitting the main known features of solar activ- ity including Maunder, Wolf minima, medieval warm period in AD and Homer minimum and Roman warm period in BC (see for details Zharkova et al, 2017).}


Second, these results with a grand cycle of about 400 years do not contradict to the 190-210 year period mentioned by Usoskin because it is, in fact, the half of the grand period of 400 years we discovered. This is the same situation as with 11 and 22 year solar cycles, which ones are valid? They both are valid, because the number of sunspots varies approximately every 11 years (from 10 to 12 in general), although the magnetic polarity fully changes over 22 years. The same happens with the grand cycle, you have their periods either about 200 years (without polarity consideration), or 400 years if the polarity of magnetic field is considered.

6. Comment 6. U17 states that he is also unclear why PZSZ17 limit themselves to the period of 1200-3000 AD? If their method worked, they could equally well predict solar activity for tens of thousands or millions of years ahead/backwards, as based on an implicit assumption of the full stationarity and perfect harmonicity of the series.

\begin{abstract}
Answer
This is a valid comments, thanks. This is exactly the direction we are working on right now. We (Zharkova et al, 2015; Popova et al, 2017a,b; Zharkova et al, 2017) take a step-by-step approach and investigate the solar activity for longer and longer periods. The authors did not limit themselves and calculated also the solar activity for 3000 year backward (to 1000 BC) (Zharkova et al, 2017), which is proven to obtain correctly the Roman's warm period and Homer grand minimum. Although, it takes some computer and researchers' time to calculate the curves for 10 millennia, or 100 millennia, nonetheless, we are encouraged by the success of the curves produced so far and will report our calculations soon.
\end{abstract}

\title{
3. Validation of the results
}

7. Comment 7. U17 clames that the result by PZSZ17 factually voids the prediction by Zharkova et al (2015) as appears obvious from their Figs.2 and 3, and the authors should have said clearly that their earlier 
results were not correct.

\begin{abstract}
Answer
We are puzzled with this statement by U17. We refer readers to the answer A1 explaining that PCA is acting as a prism on magnetic field waves allowing us to derive wavelengths of different components of the waves, which form the overall magnetic field of the Sun.

In this context, the statement by U17 is equivalent to saying that ultraviolet light is not correct compared to the white light. But this statement would be a complete nonsense, because UV light is a part of white light, similar as blue light, green light, yellow light, orange light and red light are. The concept is clear - the visible magnetic field on the surface is a superposition of many waves. And we add them one by one, until we get the closest correspondence to the observations. Our approach explans logically where the waves come from, why they produce some visible stochasticity and how by adding more wave component we arrive to the precise description of the observed solar activity. This can be done step by sten as we show it.
\end{abstract}

8. Comment 8. U17 states that even the new result by PZSZ17 disagrees with the available data for the last centuries. While the authors did not show a direct comparison between their results and other direct/ indirect data on solar activity, done by U17 in Figure 1 for decadally averaged data (modulus of the final prediction shown in Figure 3 of PZSZ17 versus different other reconstructions, based on sunspot counts/drawing and cosmogenic isotopes. While Dalton and partly Maunder minima are somehow reproduced by the PZSZ17 model, the Sporer minimum in the 15-16th century is totally missed by the method, which instead forecasts a very high activity comparable to that in the 20th century. In fact, any noisy time series with approximately the correct autocorrelation can match some of the variations purely by chance.

\title{
Answer
}

We are pleased to see that U17 admits at last that our summary curve represents very well not only Maunder, Wolf, Homer and other grand minima, but also Dalton and other centennial minima. This was the 
aim of this PZSZ17 paper and evidently it is achieved.

The disagreement of our summary curve with the alleged Sporer minimum has also caught our attention and we have submitted another paper explaining this disagreement (Zharkova et al, 2017). The paper is under the review, while the e-print is pre-published (Zharkova et al, 2017).

We elaborate in few answers below on some details of this paper explaining that Sporer minimum is, in fact, either a) the artefact of the carbon time dating technique affected by the increased radiative background caused by explosion of a very close (600-700 light years) supernova Vela Junior occurred in 1280-1290 in the Southern hemisphere or b) the consequence of very strong cosmic rays produced by this supernova, which have significantly overrriden the effects of solar activity.

9. Comment 9. U17 comments that PZSZ17 have not demonstrated that the agreement between their model and the sunspot number is better than a chance.

\begin{abstract}
Answer
We are extremely puzzled with this statement by U17, given the very good fit of sunspot numbers for cycles 21-23 shown in Fig.3 (taken from Fig. 2 in Zharkova et al, 2015) or for the naked eye sunspots observed in pre-telescope era (Fig.4 from Zharkova et al (2017)). In fact, Fig.3 clearly demonstrated that the sunspot numbers are closely reproduced by the modulus summary curve. Moreover, it showed that in cycle 23 the measured were systematically over-estimated compared to the summary curve that was later confirmed by Clette et al, 2014). However, given the fact that sunspots reflect a superposition of various waves of toroidal field, while we decompose the poloidal field waves into separate components, nobody can expect them to be identical, similar to white light emission is not identical to any of light waves of different colours.
\end{abstract}

10. Comment 10. U17 states that Sporer minimum was one of the deepest and longest grand minima of solar activity (bigger than the Maunder minimum), and its existence is beyond any doubts as follows from numerous independent results based on cosmogenic nuclides 10Be and 
14C (e.g. Beer et al, 2012; Steinhilber et al, 2012; Usoskin et al, 2017; Inceoglu et al, 2015). U17 states that he isaware of an attempt of author to demolish the Sporer minimum (Zharkova et al, 2017), but it is not yet published in a refereed journal and also contains serious flaws to be addressed beyond this Comment upon publication of that work. Thus, we have no reason to believe in the non-existence of the Sporer minimum.

\section{Answer}

Factually, this comment by U17 highlights the problem we noticed and reported in our recent paper (Zharkova et al, 2017), which is prepublished in the e-archive. As the history teaches from a comparison of Ptolemy and Copernicus models of the solar system, it does not matter what number of authors repeated the same calculations, the matter is what assumptions and methods they used. If they use the same methods and assumptions, they will reproduce the same results. Hence, the authors listed in this comment by U17 used the models similar to that by U17 and thus they obtained the similar results. While we discovered something, namely eigen vectors of solar oscillations, that the other authors obviously overlooked, despite of a number of hints from the sunspot and terrestrial features.

Given that our summary curve reproduces most of the grand minima in the past including Maunder and Wolf grand minima in $\mathrm{AD}$ as well as Homer grand minimum and Roman's warm period in $\mathrm{BC}$, we have the solid facts to believe that the summary curve describes correctly (as good as a dipole wave can do) some basic patterns of the solar activity up to $1000 \mathrm{BC}$.

However, we also noticed the major discrepancy with Sporer minimum, which cannot be reproduced by any of the additional waves or mechanisms. Our summary curve predicts a normal maximum of the grand cycle during the period of alleged Sporer minimum, contrary to prediction of a long minimum of solar activity made by the authors listed in the comment by U17. We argue (Zharkova et al, 2017) that all these predictions have two possible explanations.

The first one is related to the fact that the abundance of this carbon 
and berrilium isotopes were calculated for the standard background radiation, while the background radiation at this period was not normal at all because od th radiation from supernova Vella Junior. Libby, the founder of the radiative carbon dating method, specifically indicated (Arnold and Libby, 1949) that the background radiation is the key parameter, which must be defined for each case separately, because it can shift the time for a few hundred of years (see for details Zharkova et al, 2017).

The second possible explanation comes again from the Vella Junior supernova effect. The cosmic rays produced by this close supernova were so strong that solar magnetic field generated during the grand maximum could not protect the solar system from their effects. These strong cosmic rays of supernova Vella Junior were the main reason, which caused the terrestrial atmosphere and biosphere affected by Vella's cosmic rays to behave similarly to the periods of minima of solar activity. This corresponds to the prediction of supernova effects on the terrestrial atmosphere (see discussion in Zharkova et al, 2017, and references therein).

In order to support our suggestion, we present the following facts:

a) Terrestrial temperature during Sporer minimum (see Fig.6) taken from the paper by Usoskin et al (2005)). If Sporer minimum was indeed the solar activity minimum as U17 claims, why then the results presented in their own paper clearly shown that the terrestrial temperature during Sporer period had a well-defined and long maximum, and not minimum, as expected for solar minima?

b) Abundances of Be during the same Sporer period in Arctic and Antarctic ices (see Fig.7 taken again from Usoskin et al (2004)) also clearly show that in the North Greenland ice the Be abundance corresponds to the solar maximum period (as our curve predicts), while Be abundance in the Antarctic ice indeed shows the sign of a minimum (or increased background radiation). This strange disbalance between the abundances in the North and South ices is very likely caused by explosion of the supernova Vela Junior, which happened in 1290 at the high latitudes of the Southern hemisphere (Zharkova et al, 2017). 


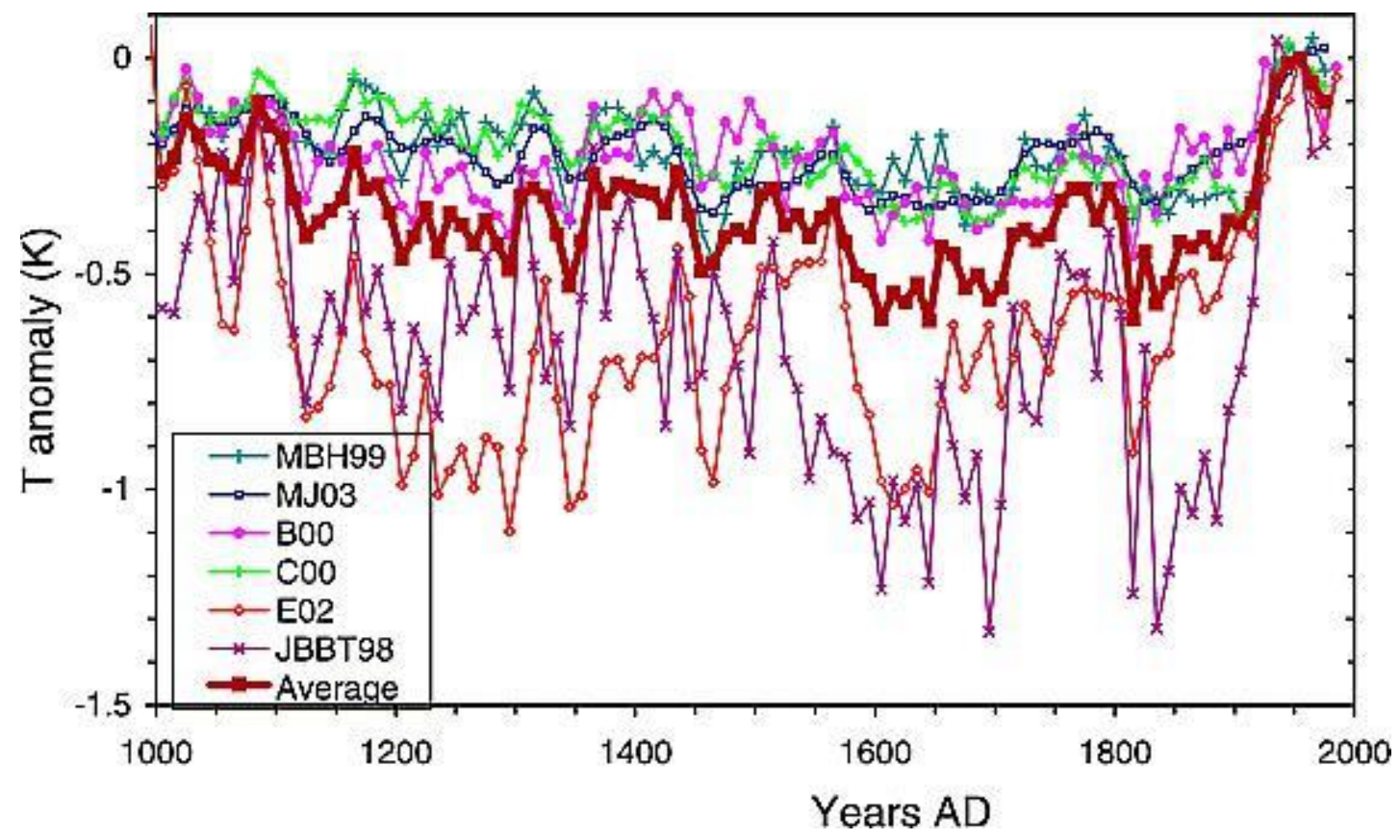

Figure 6: The terrestrial temperature variations in the past millennium derived by 7 observations with the average shown by the thick burgundy line (extracted from Fig.1 by Usoskin et al. 2005). 
Because this supernova was located in high latitudes of the Southern hemisphere, it could not be seen from the Northern hemisphere at that time and was not discovered until 20 century with use of the modern methods. Also given this southern location, the supernova's radiation and cosmic rays are very likely to affect mostly the Southern part of the Solar system and the Earth, as a part of it. This is exactly as the ice curves derived by Usoskin (see Fig.7) clearly show for increased abundances of Be in Antarctic ice, while the Northern part (for Greenland ice) this abundance corresponds to the solar maximum as it should.

c) Strongest auroras during the alleged Sporer minimum We discovered (see Zharkova et al, 2017, and references therein) that during Sporer period there were the strongest auroras ever seen in the terrestrial atmosphere. These auroras were observed for long times and often seen in the Mediterranean countries. This increased auroral activity in the Earth atmosphere is contrary to the alleged Sporer minimum when auroras must be reduced as it would normally happens during the solar minimum. During the periods of solar minima there is a little chance of generation of solar energetic particles due to the absence of any active features. The fact that during Sporer minimum powerful auroras were observed clearly indicates that these auroras can be only caused by cosmic rays coming from the outside of the solar system. At the coincidence of these auroras with the timing of the supernova Vella Junior, which keeps radiating them at much lower pace until now, indicates undoubtedly where these strong cosmic rays, or particle beams come from.

11. Comment 11. U17 states that the failure of the method in PZSZ17 to reproduce a major grand minimum of solar activity five hundred years ago invalidates any predictive capability of the model.

\begin{abstract}
Answer
This is a wrong statement because our summary curve even for dipole sources (Fig. 3 in Zharkova et al, 2015) or Fig. 2 in PZSZ17, or Fig.1 in Zharkova et al (2017) predicts a large number of the major grand minima and maxima, including Maunder, Wolf, Homer grand minima and medieval and roman warmth periods. Now, with the addition of a quadruple component in PZSZ17 and in Popova et al (2017b), we can
\end{abstract}




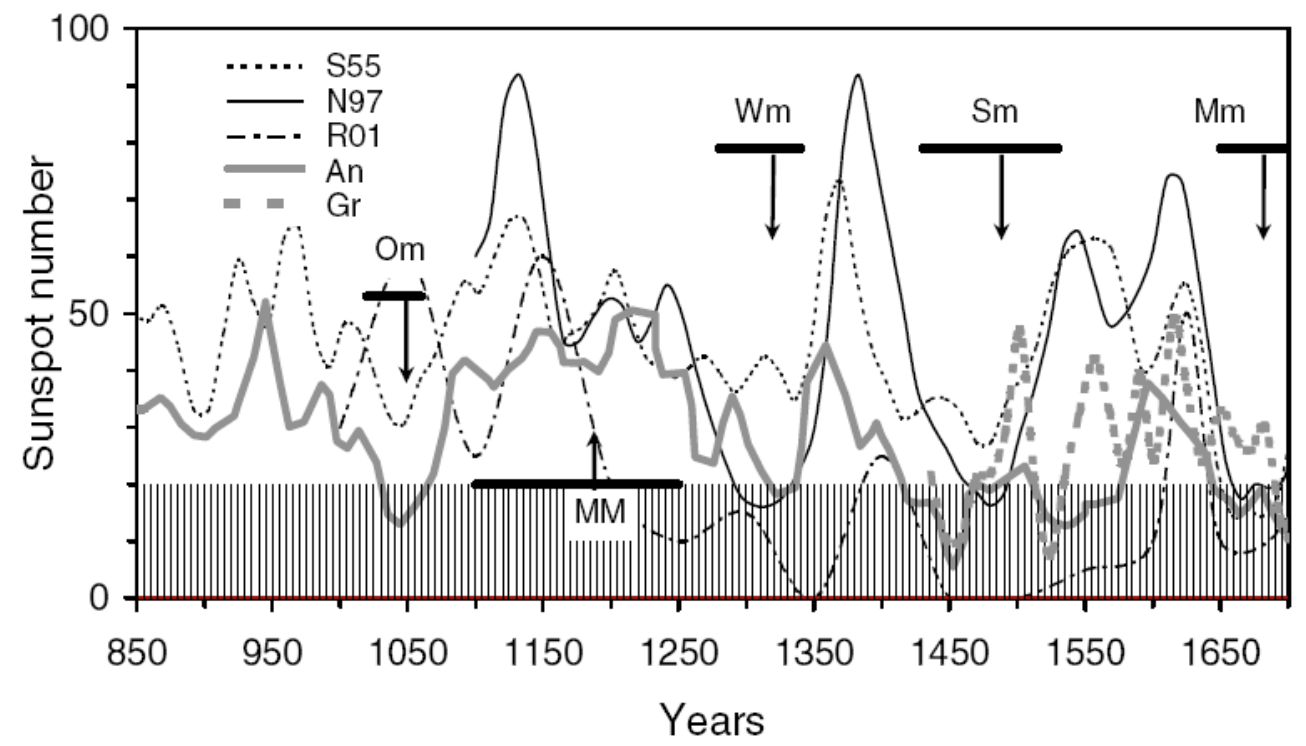

Figure 7: Reconstruction of solar activity from Greenland (Gr) and Antarctic (An) ice by Usoskin et al. (2004) (their Fig.6) with Wm indicating Wolf, Sm -Sporer and Mn Maunder minima. 
predict correctly also Dalton and other centennial minima, in addition to grand minima.

12. Comment 12. This U17 comment claims that in PZSZ17 it is not only the Sporer minimum which is not reproduced. The overall result shows no statistically significant correlation with other series. For example, the Pearson correlation coefficient (no time shift) between the Popova et al (2017a) and Muscheler et al (2016) curves, shown in Figure 1, for the period $1200-1900$ is 0.16 , which implies insignificant 2 correlation $(p \approx 0.2)$. The correlation between Popova et al (2017a) and International sunspot number v2.0 (ISN) (Clette et al., 2014) curves for the period 1700-2000 (viz. excluding the Sporer minimum) is also insignificant $(0.33, \mathrm{p}=0.16)$. For comparison, the correlation between (Muscheler et al, 2016) and ISN series for the period $1700-1900$ is highly significant $(0.64, \mathrm{p}=0.007)$. Thus, the method is unable to reproduce the observed solar variability for the last centuries, which makes any prediction from this model unreliable.

\section{Answer}

a) We are very surprised that someone can seriously consider comparing the data taken from a small plot presented in Figs. 2 or 3 in PZSZ17 with any other data. We would be concerned about any accuracy of the data taken from the small curves published in two-column text of the paper.

b) However. as we explained in the answer A1, there is no any point to compare white light (whole magnetic field/ sunspot activity) with ultraviolate light emission (dipole magnetic wave) and to declare that they are different. Yes, they are diferent by default because the while light is decomposed into 7 basic waves of different coulour and wavelegths, which are never the same as the white light wave. The same applicable to the magnetic waves on the Sun. The appearance on the solar surface reflect a combination of all magnetic waves (or white light for magnetic waves).

We have demonstrated in our papers how to decompose these waves into simpler components (or waves of different wavelengths) according to their eigen values. The first pair of these components, principal com- 
ponents, which, similar to UV light, have the strongest power. These waves are found to be produced by dipole dynamo waves, reproducing some basic features of the magnetic field (and solar activity) appearance on the surface. In order to reproduce the full picture, we need to add other waves with other eigen values (or other wavelegths in the light). This might take some time, but it is a very perspective way to work on the problem of solar activity rather than assuming its stochasticity, which condems the Sun to a close explosion.

c) Another key point is that the sever errors occur if one applies classic statistics developed for the data with normal (Gaussian) distributions to the data, which by definition do not have normal distribution at all, because they are periodic functions (see answer A1). For non-normal distributions there is a special non-parametric statistics developed (see numerous books in statistics). Therefore, use of Pearson correlation coefficient for periodic data is a serious error, the authos should use, at least, either Kendall or better Spearman's rank correlation and specific regression methods for periodic functions discussed in answer A1.

As results, any numbers found for a periodic function using the normal statistics are simply incorrect (see answer A1). This statement is proven by Usoskin own data shown in the comment showing that the Pearson correlation of 0.64 corresponds to the confidence level of $\mathrm{p}=0.007$. But this $p<<0.05$ proving without any doubts that this Pearson correlation result is statistically unsignificant (Seber, 2012).

\section{Theoretical speculations}

13. Comment 13. U17 commented: The theoretical speculations in PZSZ17 make little sense and are hardly relevant. The ad-hoc introduced quadruple component is vague. The authors wanted to add a third harmonic component to their model, but it is ungrounded why it should be a quadruple mode.

\section{Answer}

U17 contradicts to his own comment 8 where it is admited that adding the quadrule component allowed PZSZ17 to recover Dalton and other 
centennial minima.

The quadruple components are the next pair of eigen vectors found with PCA from Scree plot (see Fig.2 and answer A1). So far we did not yet derive the symbolic expressions for these two quadruple waves obtained from observations as we did for dipole waves, or principal components in Zharkova et al (2015). As we know from PCA that each of its component is likely to present simple physical mechanisms, so for the next pair of waves we use quadruple sources. Given the fact that the two PCs are reproduced very well by the dipole dynamo waves (Zharkova et al, 2015), and by looking at the cross-correlation plots with four magnetic sources (see Fig.5 and answer A1), it was logical to assume that the second pair of waves are produced by quadruple magnetic sources following the model described by Popova et al (2013) with periods slightly longer that for the waves produced by dipole sources.

This role of quadruple field was already investigated by other authors (see, for example DeRosa et al., 2012; Dikpati et al., 2016; Syukuya and Kusano, 2016, and references therein). It was also investigated by us for latitudinal variations of these waves in different cycles (Popova et al, 2013) showing that the addition of quadruple waves helped us to explain more accurately the amplitude and phase variations of latitudinal magnetic waves derived for cycles 21-23.

This addition of quadruple waves to the dipole ones allowed us to recover Dalton and other centennial minimum in the last grand cycle, which could not be seen in the previous summary curve derived from 2 PCs (or dipole magnetic waves). This indicates that we are moving in the correct direction. In the other paper (Popova et al, 2017b) we have considered Gleissberg periods from 80 to 110 years and compared the outcomes.

14. Comment 14. U17 states that PZSZ17 were unable to find this quadruple mode in the real solar magnetic data and introduce it just out of the blue.

Answer

This is a wrong comments, because we never stated in PZSZ17 that 
we have not found quadruple component in the real magnetic data. To the contrary, in PZSZ17 and in the answer A1 above we wrote that with PCA we found not only two principal components of waves generated by dipole magnetic sources, but also 3 other pairs shown in the Scree plot (Fig.1 in Zharkova et al, 2012). The second pair of waves is assigned to quadruple magnetic sources (Popova et al, 2013), these curves can generated as eigen vectors using the second pair of eigen values seen in the Scree plot (Zharkova et al, 2012) found from the original magnetic field .

What we have not yet managed to do is to find the symbolic expressions for these quadruple waves using Euriqa software, because of a lack of time and other duties. So, in order to check if the quadruple waves can improve the fit of our summary curve to the basic activity feature and based on a very good fit of the two PCs with theoretical dipole dynamo waves, we decided to use the theoretical quadruple waves (Popova et al, 2013), generated by a dynamo model, instead of observational ones, in order to test the idea if they help us to recover Dalton and other centennial minima.

The idea worked perfectly well as one can see from PZSZ17, (Popova et al, 2017b) and by the admission of U17 in comment 8 .

15. Comment 15. Another comment by U17 states that substituting the full dynamo equations with the equations for selected modes is a dangerous exercise, which can easily lead to a spurious result. In PZSZ17 the authors have not provided solid arguments that such a substitution is representative for solar activity on long-term scale. While the two main components are at least based on data (though in a non-rigorous manner), the third component has no clear meaning.

\section{Answer}

First, let us remind to U17 that the dynamo equations were derived by Parker for dipole magnetic sources and used by all theoreticians as a standard approach. As we discussed in answer A13, we added consideration of quadruple waves following DeRosa et al. (2012) and Popova et al (2013). Also the other authors (Dikpati et al., 2016; Syukuya and Kusano, 2016) after us used different modes (dipole and quadruple) of 
dynamo magnetic waves in the Sun.

Second, contrary to U17's suggestions, the combination of waves generated by different sources is the only way to investigate a particular event, like scientists did for the electromagnetic radiation of white light (see answer A1). Discovery of a glass prism and its decomposition of the white light into wavelegths allowed to develop the wave theory of geomertic optics that gave the boost to building optical instruments and led to many discoveries.

We had applied the PCA tool to decompose these magnetic waves and then, using the accumulative expertise of the solar dynamo community, we obtained a very good fit of our summary curve to the basic solar features (Zharkova et al, 2015). Now we have showed (Popova et al, 2017a,b) that by adding the next set of components of dynamo waves we managed to reproduce Dalton and other centennial minima using the classic theory of interference of waves generated by dipole and quadruple sources (see also answer A3).

\title{
5. Summary
}

16. Comment 16. U17 comment that PZSZ17 contains several flaws, which make the prediction of solar activity for the next thousands years unreliable. The method of PZSZ17 is based on an oversimplified and unreliable ad-hoc multi-harmonic representation of solar activity, and lacks quality control.

\begin{abstract}
Answer
Contrary to U17 comment, we have shown above in the answers A1A6 that the method used is the precise method of defining the eigen values and vectors of Sun's own oscillations using PCA and the most appropriate method with Hamiltonian approach for defining a periodic function with symbolic analysis. As results, our summary curve reproduces very well the solar activity in the past 800 (and 3000) years including not only grand minima (Maunder, Wolf) but also the local minima (Dalton and centennial ones). We believe that the Sun is on our side, because in a few years time our star will start the next grand
\end{abstract}


minimum (2020-2053), as we predicted and everyone on the Earth will witness it, including U17.

17. Comment 17. U17 conslusive comment states that, in particular, the background solar dataset (35 years) does not allow determination of periodicities with sufficient accuracy to justify the beating period of 400 years. It is therefore impossible to make harmonic predictions for thousands of years based on only 35 years of data.

\begin{abstract}
Answer
This is a completely unsubstatiated statement, which is not supported by any evidences presented in our papers. As explained above in answers A1-A16, we used the well-defined mathematical methods widely utilized by other physicists: principal components analysis, symbolic regression analysis and Parker's dynamo theory, which are all well described, respected and used by the other researchers. Obviously, when the correct methods are applied, it is possible to derive the parameters of own solar oscillations, or its eigen vectors, from the magnetic field data taken even from a single cycle, or from a pair of cycles, or from all three cycles considered.
\end{abstract}

18. Comment 18. The comment by U17 concludes that the result of the post-diction contradicts the observational data of the past solar activity. In particular, it fails to reproduce the greatest grand minimum of solar activity, Sporer minimum, and also does not correlate with the known variability of solar activity in a statistically significant manner.

\title{
Answer
}

This is a completely unsubstantiated statement as we shown in the answers A10-A12.

19. Comment 19. U17 concludes that theoretical speculations make little sense. In particular, the third quadruple component of the model is introduced purely ad-hoc with the purpose of obtaining a beating period of 100 years.

Answer

Theoretical simulations in PZSZ17 confirm the authors' assumptions 
where these waves come from -quadruple magnetic field. This result presented in PZSZ17 has been supported by two different referees, contrary to U17 comments. For the rest, please, see the answers A13-A15 above.

20. Comment 20. U17 statement that a multi-harmonic mathematical model, hardly related to full solar dynamo theory presented by PZSZ17, is not applicable to realistic solar conditions because of the significant chaotic/stochastic intrinsic component and strong non-stationarity of solar activity. The obtained result is apparently inconsistent with the data in the past and, thus, cannot be trusted for the future predictions.

\section{Answer}

The summary of U17 is not correct as we shown iny all the answers above. Moreover, we obtained more than 1000 various replies from various researchers all over the world about our paper (Zharkova et al, 2015) and most of them report that our results confirm their own findings in the terrestrial activity.

It looks like the only theory, which is struggling from our research presenting decomposition of solar magnetic waves into components, like the white light, is U17 own theory about stochastic solar activity. All other researchers accept this evident approach to solar activity that helps to pour the light on many other events in solar-terrestrial connection.

Moreover, everyone on the Earth should be very worried about the Sun and its longivity, if it's heartbeat, or dynamo machine, is so unpredictable and stochastic, because this is the sign of possible explosion. While our approach explain the visible irregularity of solar magnetic field by the interference effects of different components. This is a simple and straightforward model, as the real model normaly is.

We shown the results by PZSZ17 are able to account for the basic grand minima of solar activity derived from the terrestrial data as shown above (Zharkova et al, 2015; Popova et al, 2017a,b; Zharkova et al, 2017) and to help with the interpretation of the research of terrestrial temperature and 
Be isotope abundances in the Arctic and Antarctic ices observed during the alleged Sporer minimum.

\section{Acknowledgement}

The authors acknowledge with thanks that this work was initiated during the visit by EP to Northumbria University funded by the Royal Society international exchange grant. This research was also partially funded by the grant ref. 16-17-10097 of Russian Science Foundation ( PI- H. Popova).

Arnold, J.R. and Libby, W.F. (1949) Age Determinations by Radiocarbon Content: Checks with Samples of Known Age, Science, 110, 678-680.

Beer, J., McCracken, K., and von Steiger, R. (2012). Cosmogenic Radionuclides: Theory and Applications in the Terrestrial and Space Environments. Berlin: Springer.

Cadavid A. C., Lawrence J. K., McDonald D. P., Ruzmaikin A., 2005, Sol. Phys., 226, 359

Cadavid A. C., Lawrence J. K., Ruzmaikin A., 2008, Sol. Phys., 248, 247

Choudhuri A.R., Chatterjee P. and Jiang J. (2007). Predicting solar cycle 24 with a solar dynamo model. Phys. Rev. Lett., 98, 131103.

Choudhuri A.R. and Karak, B. B. (2012). Origin of Grand Minima in Sunspot CyclesPhys. Rev. Lett., 109, 171103.

Clette, F., Svalgaard, L., Vaquero, J., and Cliver, E. (2014). Revisiting the sunspot number: A 400-year perspective on the solar cycle. Space Sci. Rev., 186, 35.

Dikpati, M., Suresh, A., and Burkepile, J. (2016). Cyclic Evolution of Coronal Fields from a Coupled Dynamo Potential-Field Source-Surface Model. Sol. Phys., 291, 339.

DeRosa, M. L., Brun, A. S., and Hoeksema, J. T. (2012). Solar magnetic field reversals and the role of dynamo families. ApJ, 757, 96. 
Inceoglu, F., Simoniello, R., Knudsen, V. F., Karoff, C., Olsen, J., TurckChieze, S., and Jacobsen, B. H. (2015). Grand solar minima and maxima deduced from ${ }^{10} \mathrm{Be}$ and ${ }^{14} \mathrm{C}$ : magnetic dynamo configuration and polarity reversal. Astron. Astrophys., 577, A20.

Holappa, L.; Mursula, K.; Asikainen, T.; Richardson, I. G. (2014) Annual fractions of high-speed streams from principal component analysis of local geomagnetic activity. Journal of Geophysical Research: Space Physics, 119, 4544.

Jackiewicz, J., Serebryanskiy, A., and Kholikov, S. (2015). Meridional Flow in the Solar Convection Zone II: Helioseismic Inversions of GONG Data, ApJ, 805, 133.

Jones, C. A., Thompson, M. J.\& Tobias, S. M. (2010) The Solar Dynamo, Space Sci. Rev. , 152, 591-616..

Jolliffe I. T. (2002) .Principal Component Analysis, Book, 2nd edition. Berlin: Springer Series in Statistics.

Karak B.B. and Nandy D. (2012) Turbulent pumping of magnetic flux reduces solar cycle memory and thus impacts predictability of the Sun's activity. Astrophys. J. Lett., 761, L13.

Kremliovsky, M. (1995). Limits of predictability of solar activity. Solar Phys., 159, 371 .

Lawrence, J. K., Cadavid, A. and Ruzmaikin, A., (2004) Principal Component Analysis of the Solar Magnetic Field I: The Axisymmetric Field at the Photosphere. Solar Phys., 225, 1.

Muscheler, R., Adolphi, F., Herbst, K., and Nilsson, A. (2016). The 140 Revised Sunspot Record in Comparison to Cosmogenic Radionuclide-Based Solar Activity Reconstructions. Solar Phys., 291, 3025.

Ogurtsov, M., Nagovitsyn, Y., Kocharov, G., and Jungner, H. (2002). Long period cycles of the sun's activity recorded in direct solar data and proxies. Solar Phys., 211, 371.

Parker, E. N., (2010). Hydromagnetic Dynamo Models, Astrophys. J. , 122, 293. 
Parker, E. N. (1993). A solar dynamo surface wave at the interface between convection and nonuniform rotation, Astrophys. J. , 408, 707.

Petrovay, K. (2010). Solar Cycle Prediction. Living Rev. Solar Phys., 7, 6.

Popova, E., Zharkova, V. V., and Zharkov, S. I. (2013) Probing latitudinal variations of the solar magnetic field in cycles 21-23 by Parker's TwoLayer Dynamo Model with meridional circulation. Annales Geophysicae, 31, 2023.

Popova, E., Zharkova, V. V., Shepherd, S. J., and Zharkov, S. I. (2017a) On a role of quadruple component of magnetic field in defining solar activity in grand cycles. J. Atmosph. Solar-Terr. Phys., doi.org/10.1016/j.jastp.2017.05.006.

Popova, E., Zharkova, V. V., Shepherd, S. J., and Zharkov, S. I. (2017b) On a role of quadruple component of magnetic field in defining solar activity in grand cycles. Proceedings of Ninth Workshop 'Solar Influences on the Magnetosphere, Ionosphere and Atmosphere', Sunny Beach, Bulgaria, May 30 - June 3, 2017, 34.

Schmidt, M. and Lipson, H. (2009) Distilling free-form natural laws from experimental data. Science, 324, 81.

George A. F. Seber, and Alan J. Lee (2012). Linear Regression Analysis, Weley series on Probability and Statistics, Wiley and Sons.

Shepherd S.J., Zharkov S.I. and Zharkova V.V. (2014). Prediction of solar activity from solar background magnetic field variations in cycles 21-23. Astrophys. J., 795, 46.

Steinhilber, F., Abreu, J., Beer, J., Brunner, I., Christl, M., Fischer, H., Heikkilae, U., Kubik, P., Mann, M., McCracken, K., Miller, H., Miyahara, H., Oerter, H., and Wilhelms, F. (2012). 9,400 years of cosmic radiation and solar activity from ice cores and tree rings. Proc. Nat. Acad. Sci. USA, 109, 5967 .

Syukuya, D. and Kusano, K. (2016). Simulation Study of Hemispheric PhaseAsymmetry in the Solar Cycle. arXiv, 1612.03294 
Usoskin, I. G. (2008). A History of Solar Activity over Millennia. Living Rev. Solar Phys., 14, 3.

Usoskin, I. (2017). Comment on the paper by Popova et al. On a role of quadruple component of magnetic field in defining solar activity in grand cycles, J. Atmosph. Solar-Terr. Phys., in press.

Usoskin, I. G., Hulot, G., Gallet, Y., Roth, R., Licht, A., Joos, F., Kovaltsov, G. A., Thebault, E., and Khokhlov, A. (2014). Evidence for distinct modes of solar activity. Astron. Astrophys., 562, L10.

Usoskin, I. G., Mursula, K., Solanki, S., Schlussler, M., and Alanko, K. (2004). Reconstruction of solar activity for the last millennium using ${ }^{10} \mathrm{Be}$ data. Astron. and Astrophys., 413, 745.

Usoskin, I. G., Schlussler, M., Solanki, S. K., and Mursula, K. (2005). Solar activity, cosmic rays, and Earth's temperature: A millennium-scale comparison. Journal of Geophysical Research (Space Physics), 110, A10102.

Vecchio, A., Lepreti, F., Laurenza, M., Alberti, T., and Carbone, V. (2017). Connection between solar activity cycles and grand minima generation. Astron. Astrophys, $\mathbf{5 9 9}$, A58.

Zhao J., Bogart R.S., Kosovichev A.G., Duvall T.L., Jr. and Hartlep T. (2013). Detection of equatorward meridional flow and evidence of doublecell meridional circulation inside the Sun. Astrophys. J. Lett., 774, L29.

Zharkova, V. V.; Zharkov, S. I. (2010). Active Latitudes for Sunspot Occurrences in the Solar Cycle 23. Advances in Geosciences, 21, 289.

Zharkova, V. V., Shepherd, S. J., and Zharkov, S. I. (2012). Principal component analysis of background and sunspot magnetic field variations during solar cycles 21-23. Mon. Notices of RAS, 424, 2943.

Zharkova, V. V., Shepherd, S. J., Popova, E., and Zharkov, S. I. (2015). Heartbeat of the Sun from Principal Component Analysis and prediction of solar activity on a millenium timescale. Sci. Rep., 5, 15689.

Zharkova, V. V., Shepherd, S. J., Popova, E., and Zharkov, S. I. (2017). Reinforcing the double dynamo model with solar-terrestrial activity in the past three millennia. arXiv, 1705.04482. 